\title{
Experimental Research of Dynamic Response of Laser-Induced Film-Substrate System
}

\author{
Baoyi Cheng1, Aixin Feng, ${ }^{1,2}$, Jiacheng Mao', Yupeng Cao1,3, Hao Wu${ }^{1}$, Xuhua Zhuang1, \\ Ziqiang Li ${ }^{1}$, Huang Y $\mathbf{u}^{1}$ \\ ${ }^{1}$ School of Mechanical Engineering, Zhenjiang, China \\ ${ }^{2}$ School of Techtronic Engineering, Wenzhou University, Wenzhou, China \\ ${ }^{3}$ School of Mechanical Engineering, Nantong University, Nantong, China \\ Email: chengbao5470@qq.com, aixfeng@126.com
}

Received 7 March 2016; accepted 25 March 2016; published 28 March 2016

Copyright (C) 2016 by authors and Scientific Research Publishing Inc.

This work is licensed under the Creative Commons Attribution International License (CC BY). http://creativecommons.org/licenses/by/4.0/

(c) $\underset{\mathrm{EY}}{\mathrm{Br}}$ Open Access

\section{Abstract}

Film-substrate's interfacial bonding strength is closely related to film quality. An excellent interfacial bonding strength is the premise for the well use of film. The laser detecting technique of discrete scratches based on laser shockwave effect is a new method, which can measure interfacial bonding strength. With this technique, film-substrate system is of transient load of different laser energy, the relation between the dynamic response characteristics of such film-substrate system and film-substrate's interfacial bonding strength is a core problem to be solved urgently. On this basis, this paper conducted research on the dynamic response characteristics of film-substrate system during laser loading process using detecting technique of PVDF patch sensor. Results show that under the irradiation of different laser energy, it can detect dynamic responses of theory models of different film-substrate system using PVDF patch sensor, wherein shockwave dynamic response and dynamic strain response are included. Laser energy and interfacial bonding strength are of a regular influence to the dynamic response of film-substrate system theory model.

\section{Keywords}

Film-Substrate System, Laser Shockwave, Dynamic Response, Interfacial Bonding Strength, PVDF

\section{Introduction}

The laser detecting technique of discrete scratches is developed on the basis of laser spallation and laser scratch

How to cite this paper: Cheng, B.Y., Feng, A.X., Mao, J.C., Cao, Y.P., Wu, H., Zhuang, X.H., Li, Z.Q. and Yu, H. (2016) Experimental Research of Dynamic Response of Laser-Induced Film-Substrate System. Journal of Materials Science and Chemical Engineering, 4, 42-53. http://dx.doi.org/10.4236/msce.2016.43006 
[1]-[4]. It changes the film-substrate system by utilizing the pulsed laser shockwave effect. The induced highvoltage shockwave (stresswave) transmits to the internal film-substrate system and a dynamic respond between the interface of film-substrate system and internal materials occurs, while the detected dynamic signals can reflect the intrinsic bonding strength of dynamic interface. Delving into dynamic response characteristics of filmsubstrate system under irradiation of pulsed laser could monitor the propagation rule of shockwave and filmsubstrate interface effect at the same time, which was conductive to revealing the failure mechanism of film-substrate system.

The researches on detection of interfacial bonding performance and measurement of interfacial bonding strength by using the laser shockwave effect can date back to the 90s. American scientists Gupta et al. [5] measured the interface tension strength between two reinforced ceramic composites in 1992 and at the same time, ABAQUS software was also adopted for simulation of elastic wave propagation and for process of transient heat transfer. Interiorly, He Pengfei et al. [6] from Shanghai Jiaotong University probed into microscopic damages of composite microstructures under the irradiation of pulsed laser in 1994, explored the correlation between failure mechanism of interfacial bonding strength and interface cracking strength, proposed the laser detecting technique of discrete scratches based on above scientific achievements and made some progress. Shi Fen [7] and Shi Fen et al. [8] delved into stress-strain characteristics of film-substrate system to concretely analyze the filmsubstrate's interfacial bonding status and influence rule on dynamic system strain by laser energy. Besides, an experimental research on the distribution law for residual stress of film-substrate system was also carried out. The complexity of stress on the surface of laser-induced materials not only reflects on the effect of laser shockwave, but also on the comprehensive non-linear superimposed result of laser shockwave effect, laser thermal effect and thermal effect.

This paper detected the laser shockwave by bonding PVDF on the back of matrix based on the established theoretical film-substrate system and delved into both the laser energy and the influence on dynamic respond of film-substrate system by interfacial bonding strength. At this time, the detection results are free from the effect of laser thermal and thermal stress without any complex consideration, which has provided a reference to reveal the failure mechanism of film-substrate system.

\section{Experimental Methods and Materials}

This experiment adopted the widely used AZ31B magnesium alloy as matrix and 316 stainless steel thin-foil as coating, wherein the thickness of film is $0.02 \mathrm{~mm}$. besides, matrix and film were bonded by different bonders, which can represent different interfacial bonding strength due to the difference in viscosity. Based on these, a theoretical model of film-substrate system was established to replace the actual model. The main performance indexes of AZ31B magnesium alloy are shown in Table 1 and chemical composites of 316 stainless-steel are shown in Table 2. Before this experiment, cutting lines of AZ31B magnesium alloy were disposed into 12 samples sized $40 \mathrm{~mm} \times 40 \mathrm{~mm} \times 1.5 \mathrm{~mm}$, polished by $100 \#-800 \#$ abrasive paper for metallograph, cleaned by alcohol and dried by cold-blast air to get samples with thickness of $0.5 \mathrm{~mm}$. This experiment established three film-substrate interface models based on the difference in bonding strength of bonders. (1) Model A: using the water layer to bond 316 stainless-steel foil and AZ31B magnesium alloy; (2) Model B: using 502 glue to bond 316 stainless-steel foil and AZ31B magnesium alloy; (3) Model C: using a double-sided tape to bond 316 stainless-steel foil and AZ31B magnesium alloy. There are respectively 4 samples in each model with a successively increased interfacial bonding strength. Besides, this experiment should ensure the good interfacial bonding status in the process of model construction and avoid vacuoles on contact surface. A black tape with thickness of $200 \mu \mathrm{m}$ was bonded to surfaces of 316 stainless-steel foil as absorbed layer before being shocked by laser. Such a black tape is sized $3 \mathrm{~mm} \times 3 \mathrm{~mm}$ and directed to the center of laser spot. The thickness and size of PVDF patch sensor are respectively $30 \mu \mathrm{m}$ and $5 \mathrm{~mm} \times 5 \mathrm{~mm}$. PVDF patch sensor was bonded on the back of AZ31B magnesium alloy and directed to laser shock areas, wherein two ports of sensor are parallel resists with the resistance value of $50 \Omega$. The concrete layout is shown in Figure 1 .

This experiment adopted YAG SGR-series pulsed laser (LSP parameters are shown in Table 3) and DL9140 digital oscilloscope with the frequency bandwidth of $1 \mathrm{GHZ}$ and maximum sampling rate of 5GS/s to collect dynamic signals detected by the patch sensor. The photodiode triggered oscilloscope to record piezoelectric signals detected by PVDF patch sensor when laser signals were detected. The four samples of each film-substrate system model were shocked once by laser with energy of $400 \mathrm{mj}, 600 \mathrm{mj}, 800 \mathrm{mj}$ and $1000 \mathrm{mj}$. The concrete pulsed LSP parameters are shown in Table 3. 


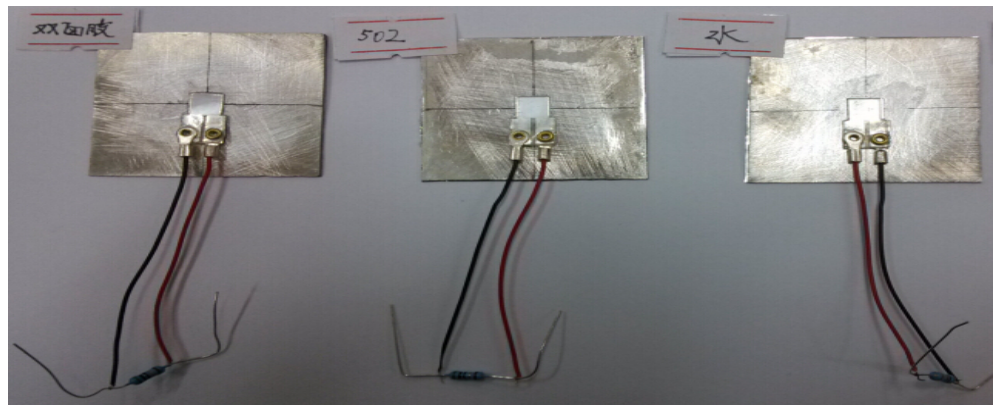

Figure 1. Layout of PVDF patch sensor.

Table 1. Main performance indexes of AZ31B magnesium alloy.

\begin{tabular}{ccc}
\hline Density $\rho_{0}\left(\mathrm{~kg} / \mathrm{m}^{3}\right)$ & Poisson's ratio $\mu E(\mathrm{MPa})$ & elastic modulus \\
\hline 1780 & 0.33 & 45,000 \\
\hline
\end{tabular}

Table 2. Chemical composites of 316 stainless steel (\%) [6] [7].

\begin{tabular}{ccccccc}
\hline $\mathrm{C}$ & $\mathrm{Si}$ & $\mathrm{Mn}$ & $\mathrm{P}$ & $\mathrm{Ni}$ & $\mathrm{S}$ & $\mathrm{Cr}$ \\
$\leq 0.08$ & $\leq 1.0$ & $\leq 2.0$ & $\leq 0.035$ & $10.0-14.0$ & $\leq 0.03$ & $16.0-18.5$ \\
\hline
\end{tabular}

Table 3. LSP parameters.

\begin{tabular}{ccccc}
\hline Shock point & Energy $(\mathrm{mJ})$ & Power density $\left(\mathrm{GW} / \mathrm{cm}^{2}\right)$ & Wavelength $(\mathrm{nm})$ & Pulse width (ns) \\
\hline 1 & 400 & 1.82 & & \\
2 & 600 & 2.73 & 1064 & 7 \\
3 & 800 & 3.64 & & 2 \\
4 & 1000 & 4.55 & & \\
\hline
\end{tabular}

\section{Experimental Results and Analysis}

\subsection{Influence on Dynamic Response Characteristics of Film-Substrate System by Laser Energy}

a. Delving into the influence on dynamic response characteristics of shockwave by laser energy

Figures 2-4 are voltage signals of model A, B and C under different laser energy. Figure 5 is pressure curve of shockwave after transformation.

The following rules can be observed form the figures:

(1) The larger laser energy is in each film-substrate system model, the stronger detected piezoelectric and shockwave signals and larger vibration amplitude would be. As shown in Figure 2(a), under the effect of 1000 mj laser energy, the highest amplitude value of shockwave piezoelectric signal can up to $2.9 \mathrm{~V}$ and the highest amplitude value of shockwave pressure can up to 9 Mpa. However, as shown in Figure 2(d), under the effect of $400 \mathrm{mj}$ laser energy, the highest amplitude value of shockwave piezoelectric signal can only up to $1.3 \mathrm{~V}$ and the highest amplitude value of shockwave pressure can only up to $3.8 \mathrm{MPa}$.

(2) Larger laser energy contributed to a longer time of system equilibrium state and the whole process from the signal detection to the eventual system stability was finished in microseconds.

The analysis suggested that, if strong-pulsed laser-induced shockwave passes through the film at a certain velocity and transmits in depth direction, reflection and transmission phenomena may occur on shockwave at the Film-substrate interface [9]. If the film-substrate interfacial bonding strengths are the same, then the energy proportion of reflection wave and transmission wave can be deemed as the same. Hence, the larger the laser energy is, the more transmission waves transmitted to the internal matrix via film, the more obvious extrusion effect between particles of matrix materials, the stronger the piezoelectric signals detected by PVDF and the larger amplitude of fluctuation would be. After these changes, the back and forth transmission of shockwave in the film-substrate system has led to the continuous change of detected dynamic response curve. The shockwave 


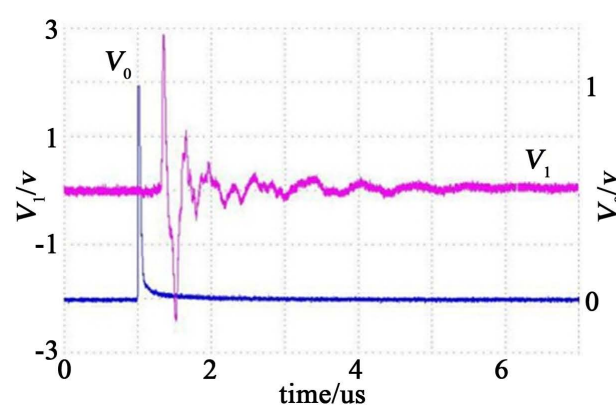

(a)

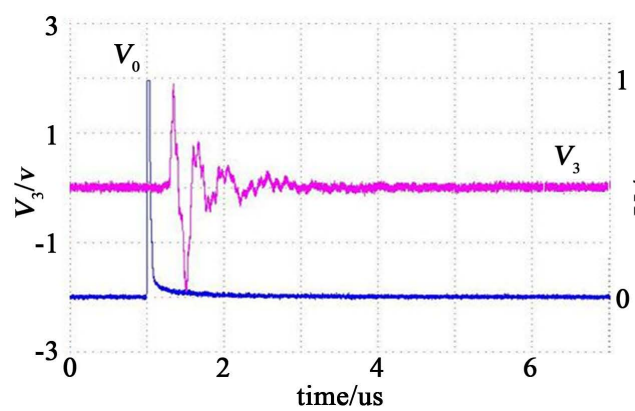

(c)

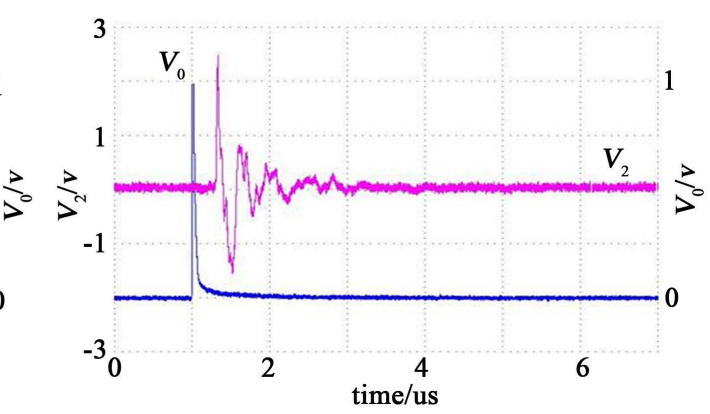

(b)

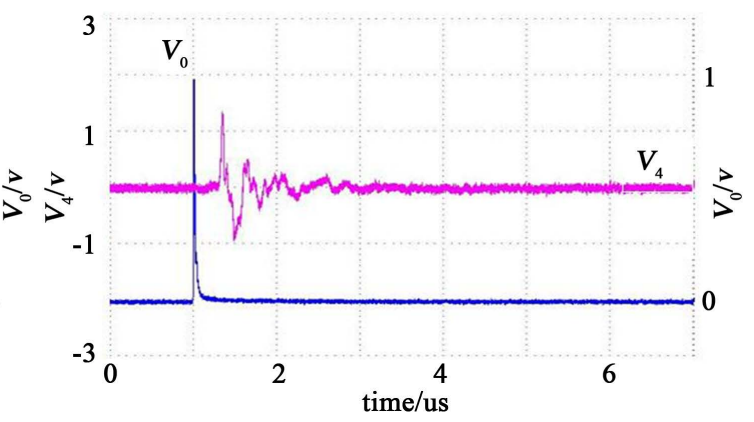

(d)

Figure 2. Voltage signal of A model under different laser energy: (a) 1000 mj laser energy; (b) 800 mj laser energy; (c) 600 mj laser energy; (d) 400 mj laser energy.

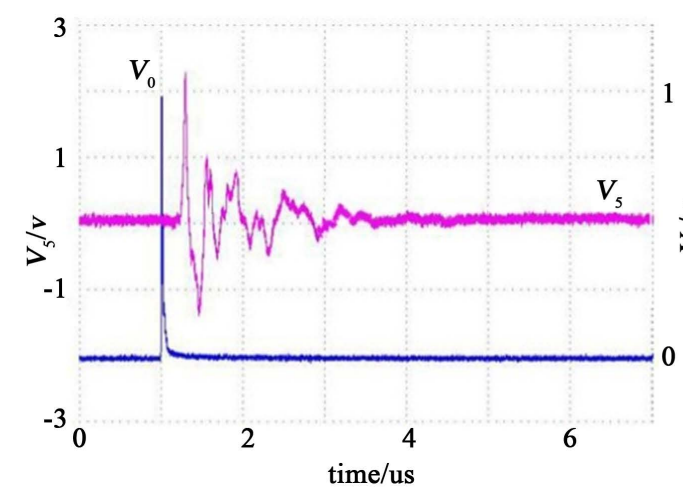

(a)

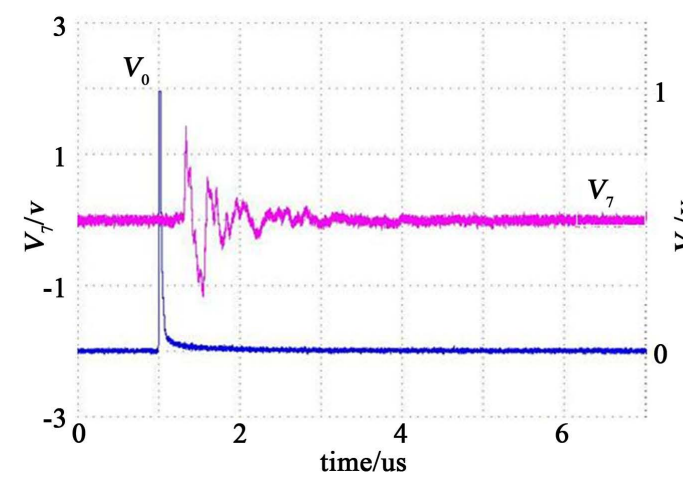

(c)

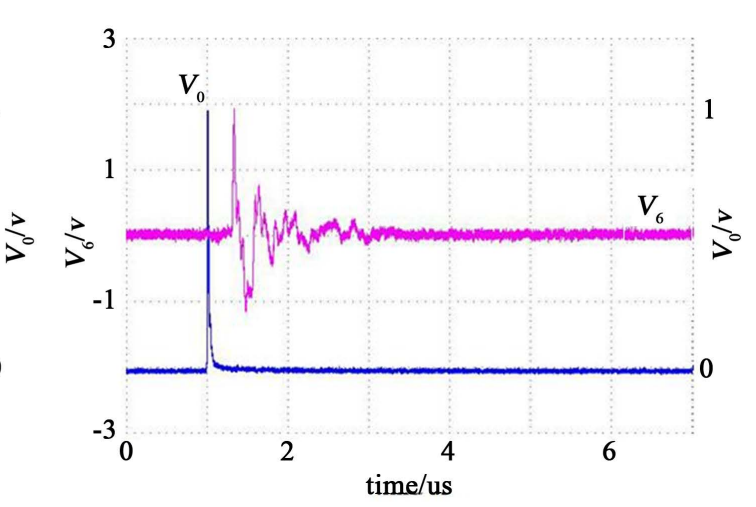

(b)

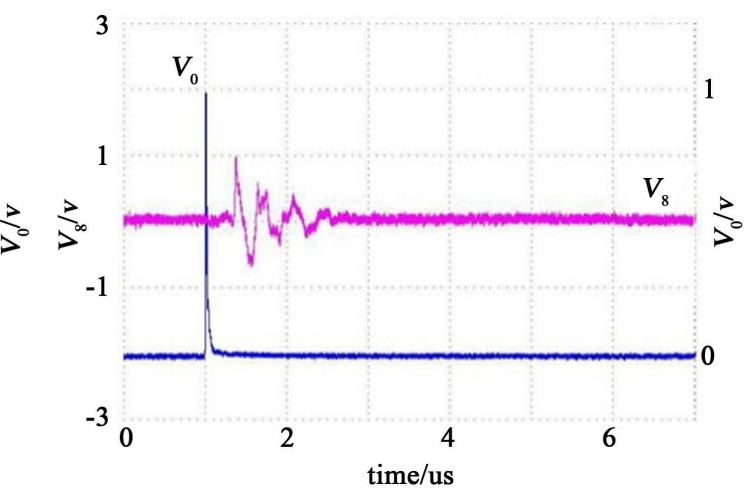

(d)

Figure 3. Voltage signal of B model under different laser energy: (a) 1000 mj laser energy; (b) 800 mj laser energy; (c) 600 mj laser energy; (d) 400 mj laser energy. 


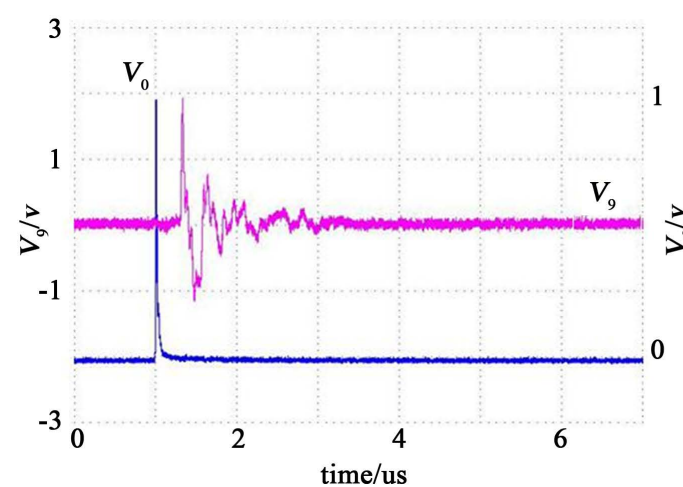

(a)

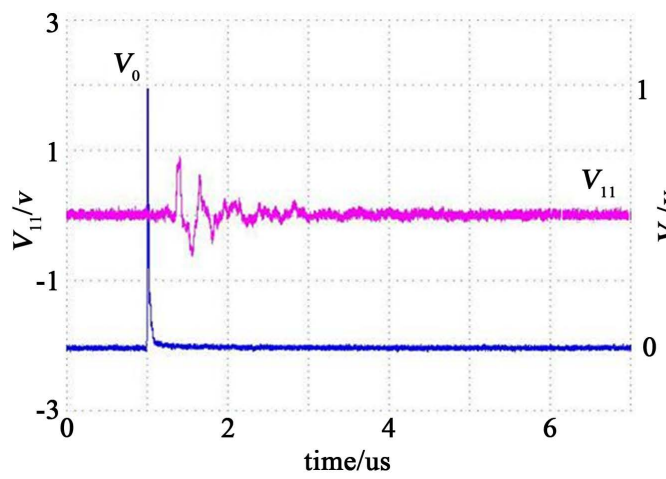

(c)

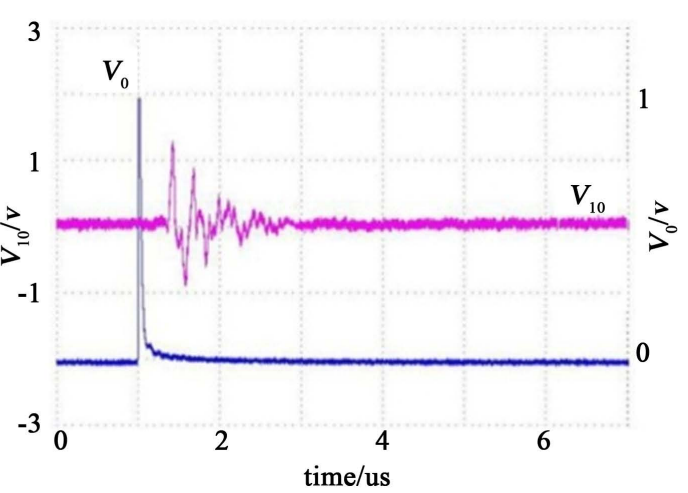

(b)

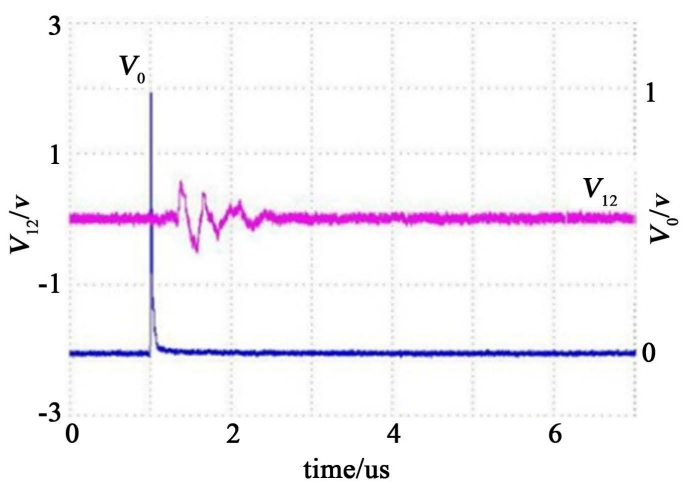

(d)

Figure 4. Voltage signal of C model under different laser energy: (a) 1000 mj laser energy; (b) 800 mj laser energy; (c) 600 mj laser energy; (d) 400 mj laser energy.

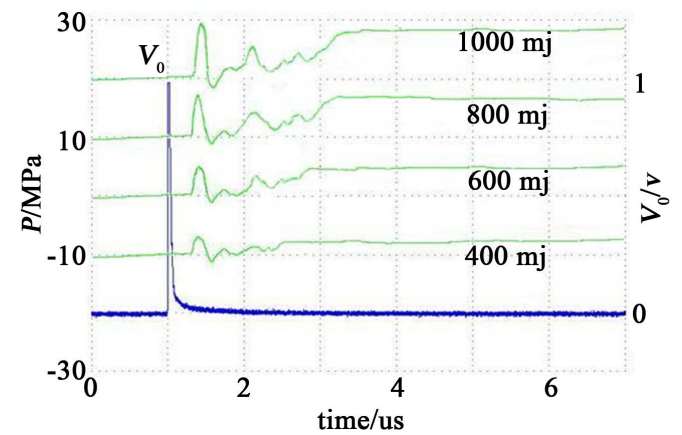

(a)

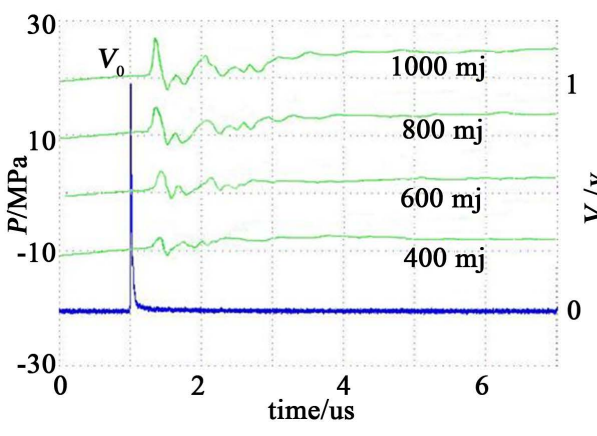

(b)

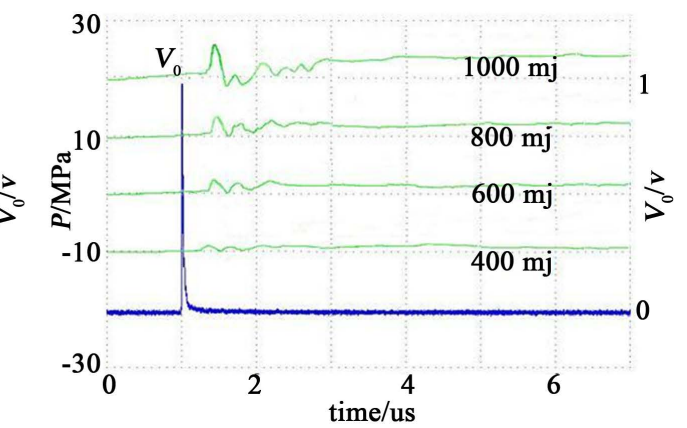

(c)

Figure 5. Pressure curve of three kinds of model under different laser energy: (a) A model; (b) B model; (c) C model. 
may have reflection and superposition phenomenon on the free surface of film and matrix in the transmission period, while reflection, refraction, transmission and superposition phenomenon occurs at film-substrate interface. The larger the energy is, the more intense dynamic response of these phenomena would be and the longer time for reaching up to the system equilibrium state would spend. Due to movement inertia of material particles and counter-acting force between particles, the eventual quiescent time of structural response would be far beyond the laser pulse bandwidth [10] and the dynamic period may remain for microseconds.

b. Delving into the influence on dynamic strain response characteristics o by laser energy

PVDF piezoelectric film can perceive crosswise and lengthwise piezoelectric effect. The crosswise piezoelectric effect refers to stretching vibration effect of film surface that is parallel to piezoelectric materials, which is represented by $d_{31}, d_{32}$. The lengthwise piezoelectric effect refers to the vibration effect of material particle that is perpendicular to the film surface, which is represented by $d_{33}$. The charge output on upper and lower surfaces of PVDF piezoelectric film satisfies the following relation [11]:

$$
Q=\sum d_{3 j} E_{P V D F} \varepsilon_{j} S \text {. }
$$

In this equation, $\varepsilon_{j}$ is the strain $(j=1-3), d_{3 j}$ is the strain constant $(j=1-3, \mathrm{C} / \mathrm{N})$, EPVDF is elasticity modulus of PVDF film $\left(\mathrm{N} / \mathrm{m}^{2}\right)$ and $\mathrm{S}$ is the area of PVDF film $\left(\mathrm{m}^{2}\right)$.

PVDF patch sensor in this experiment was bonded on the back of sample which directs to the center of laser shock area, so the dynamic response of materials particles perpendicular to the film surface were detected during the laser shock process, wherein the crosswise vibration effect can be neglected. Hence, it can be simplified as the following equation [12]:

$$
Q=\mathrm{d}_{33} \varepsilon_{3} E_{P V D F} S .
$$

For quantity of electric charge transmitted by the oscilloscope during the collecting dynamic process, the following equation is met between voltage signal $V(t)$ and transmitted quantity of electric charge $Q(t)$ at time $t$ :

$$
Q(t)=\int_{0}^{t} \frac{V(t)}{R} \mathrm{dt} .
$$

This paper united Equations (2.2) and (2.3) and put in voltage signal $V(t)$ detected by oscilloscope to acquire curve of $-t$. Figure 6 is the dynamic strain curve of film-substrate system under different laser energy.

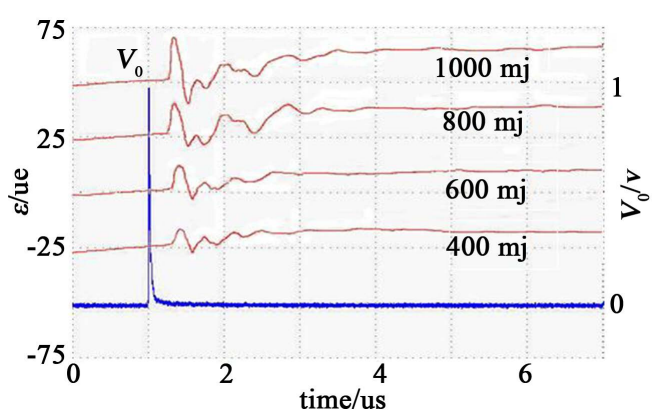

(a)

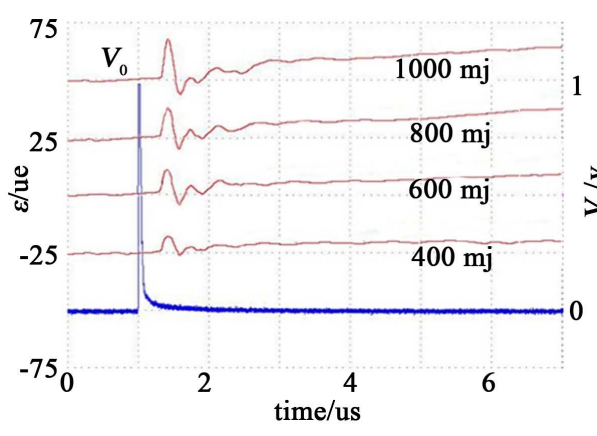

(b)

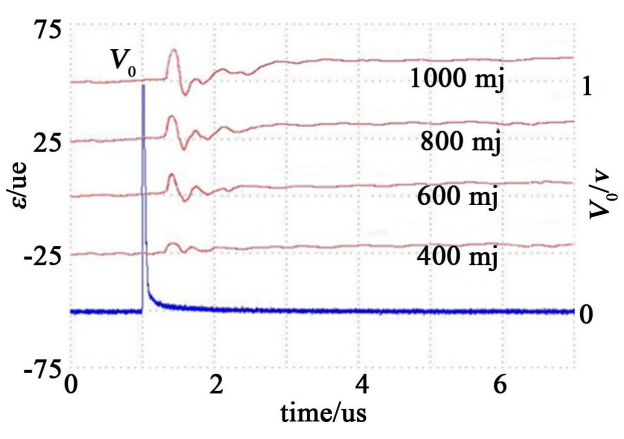

(c)

Figure 6. Dynamic strain curve of three kinds of models under different laser energy: (a) A model; (b) B model; (c) C model. 
The following rule can be observed from Figure 6: PVDF detected the stretching strain at first, then the stretching strain gradually lessened and the compression strain occurred along with a continuous change; the larger the laser energy is, the larger peak value of stretching strain and stretching strain of eventually generated after the system quiesce would be.

Based on the analysis, the reason why PVDF detecting stretching strain at first is that laser-induced shockwave transmission effects upon the back of sample. The compression wave achieved the mutual extrusion of material particles, so protuberant distortions of a certain amount were even generated on the back of sample and the stretching strain was detected. The reflection springback of compression waves formed stretching waves [13] and stretching strain was then released to form into compression strain during the pressure uploading period, making the strain curve transform from stretching strain to compression strain. If the laser energy is enlarged, those high-energy stress waves may tighten the compression between material particles. Therefore, the dynamic behaviors would be more severe, the curve would be more fluctuant and the peak value of stretching strain would be larger. Thanks to the attenuation of energy, the reciprocal conversion between the stretching strain and compression strain eventually reached to a status of equilibrium and the accumulative stretching strain effect would also enlarge the amount of stretching strain generated by the system.

\subsection{Influence on Dynamic Response Characteristics of Film-Substrate System by Interfacial Bonding Strength}

a. Delving into the influence on dynamic response characteristics of shockwave by interfacial bonding strength

Figures 7-10 are voltage signals of film-substrate system under 3 interfacial bonding strengths of laser effect. Figure 11 is pressure curve of shockwave after the transformation of equation.

The following rules can be observed form the figures:

(1) If the laser energy effects are the same, the larger the interfacial bonding strength is, the smaller the peak values of piezoelectric and dynamic pressure signals would be. The maximum peak values of piezoelectric and

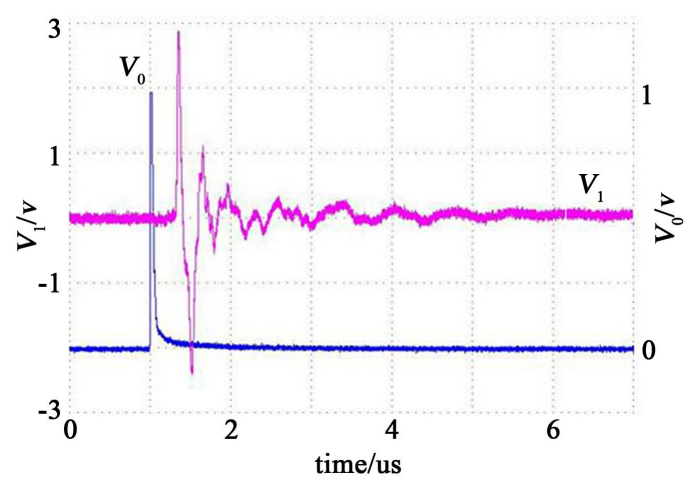

(a)

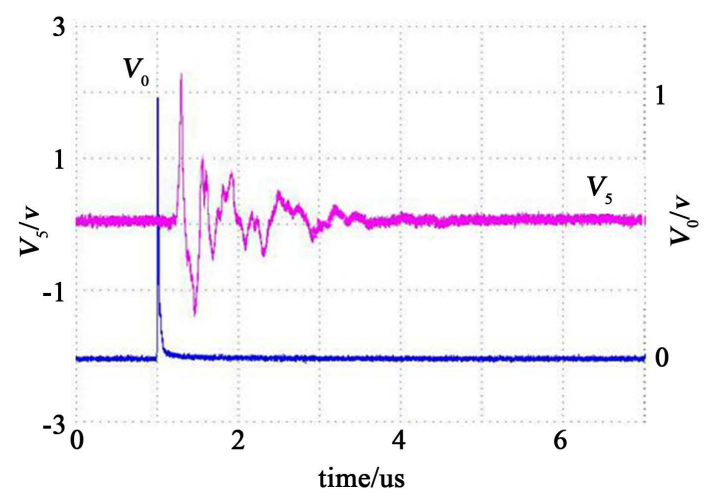

(b)

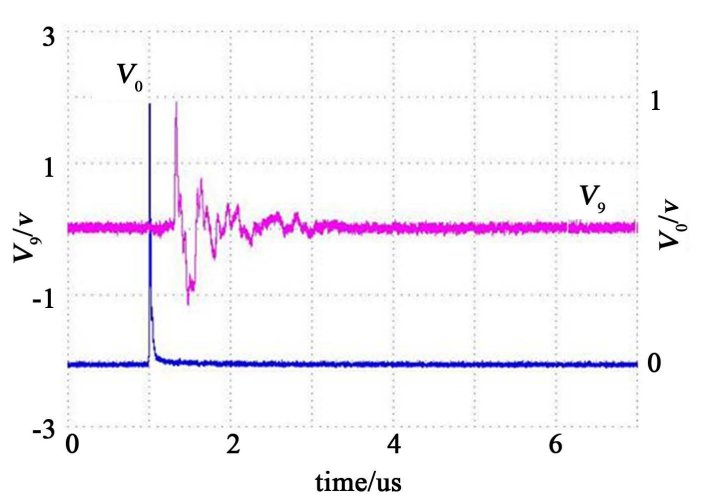

(c)

Figure 7. Voltage signal of three models under 1000 mj laser energy: (a) A model; (b) B model; (c) C model. 


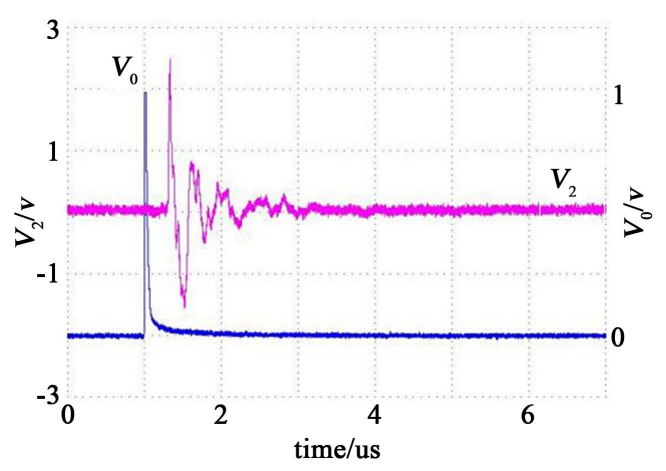

(a)

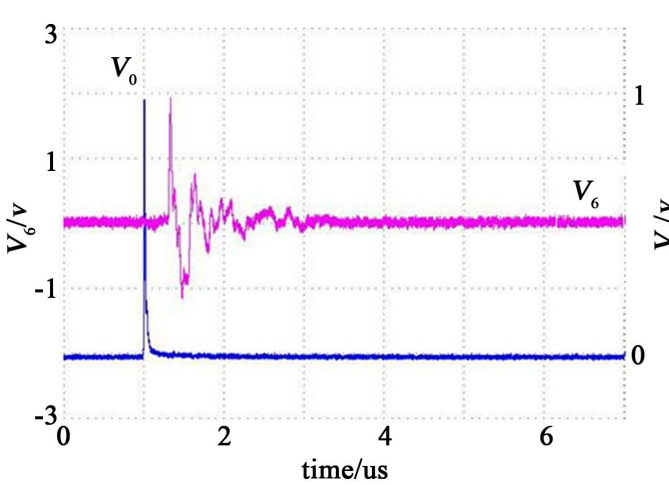

(b)

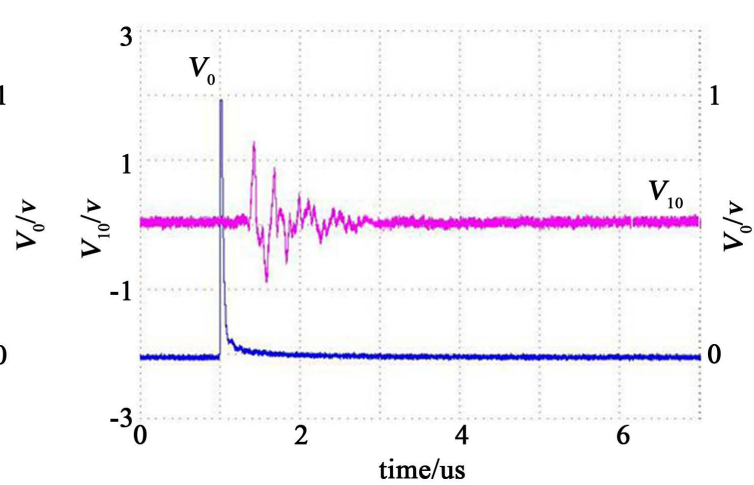

(c)

Figure 8. Voltage signal of three models under 800 mj laser energy: (a) A model; (b) B model; (c) C model.

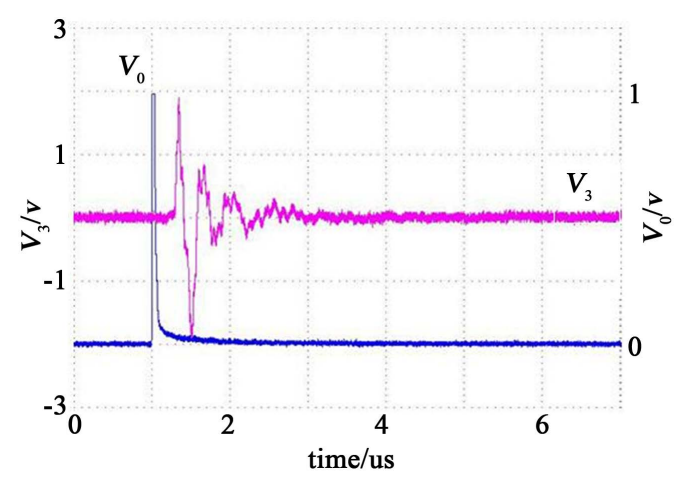

(a)

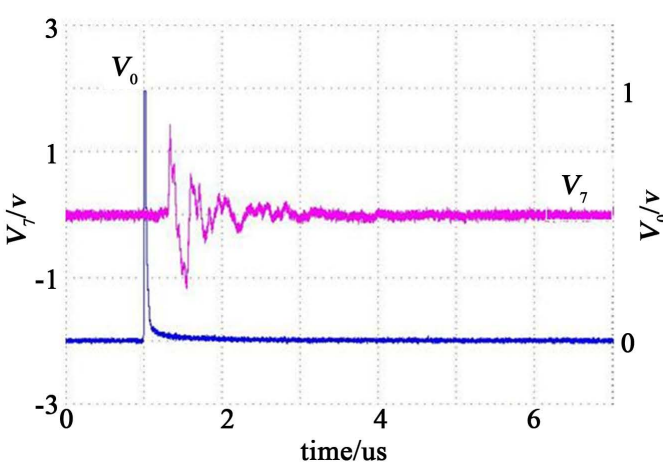

(b)

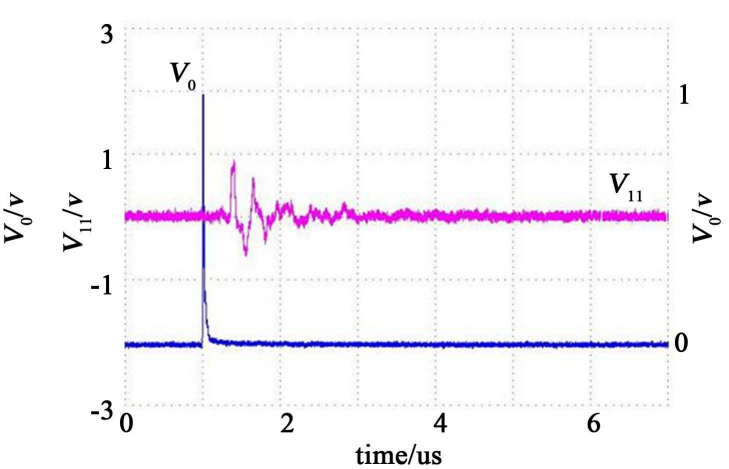

(c)

Figure 9. Voltage signal of three models under 600 mj laser energy: (a) A model; (b) B model; (c) C model. 


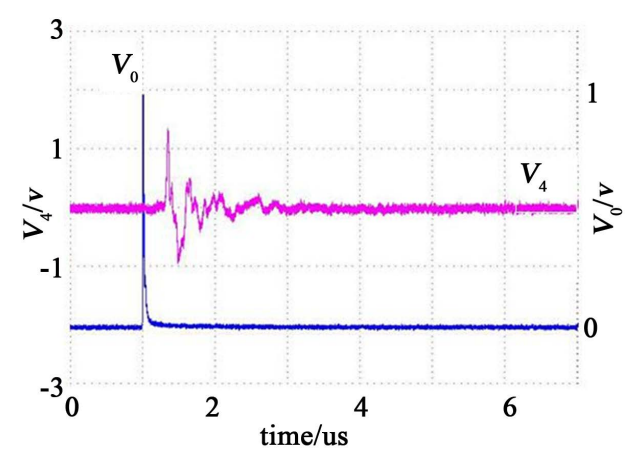

(a)

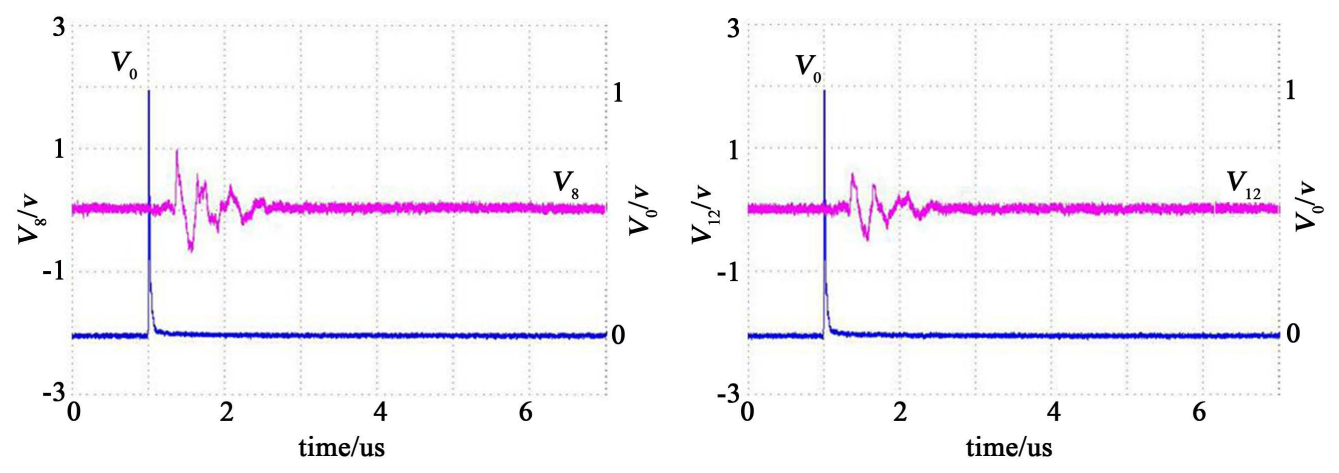

(b)

(c)

Figure 10. Voltage signal of three models under 400 mj laser energy: (a) A model; (b) B model; (c) C model.

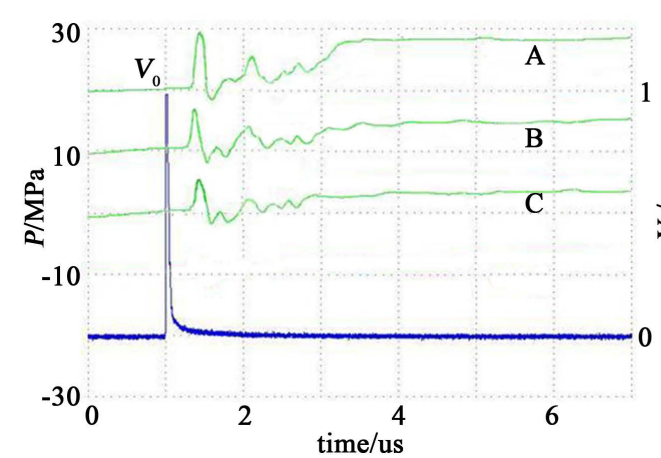

(a)

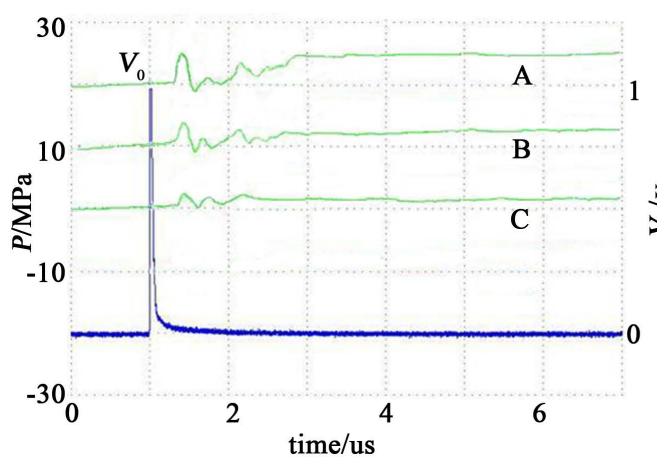

(c)

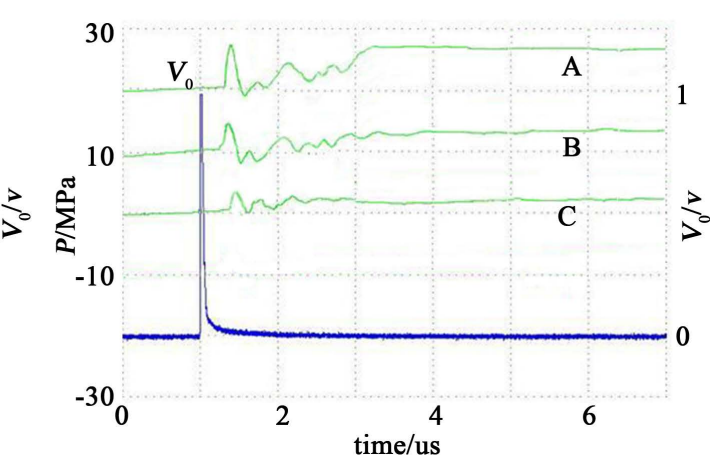

(b)

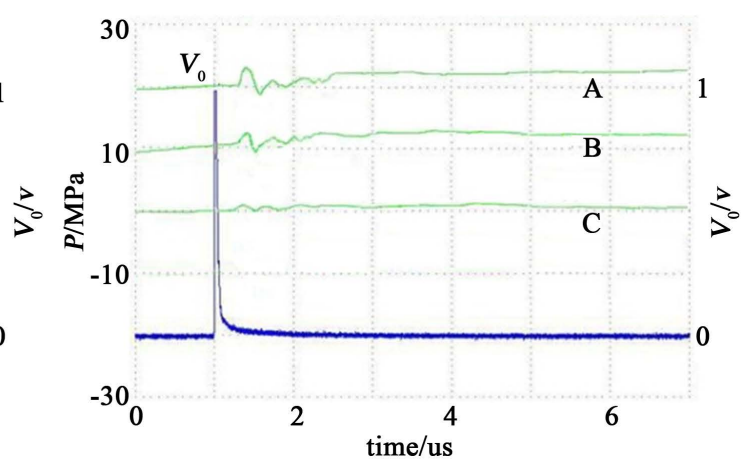

(d)

Figure 11. Pressure curve under different interfacial bonding strength: (a) 1000 mj laser energy; (b) 800 mj laser energy; (c) $600 \mathrm{mj}$ laser energy; (d) $400 \mathrm{mj}$ laser energy. 
dynamic pressure signals detected by PVDF occurred in model A, B was followed by A and C had the minimum peak values of piezoelectric and dynamic pressure signals.

(2) The piezoelectric wave curve detected in model A is much smoother with little small-amplitude fluctuation embossment, while model $\mathrm{C}$ had the least smooth piezoelectric wave curve. Judging from the pressure curve, model A needed the most time to reach the equilibrium state and dynamic process, while model $\mathrm{C}$ needed the least time and model B fell in between the two.

The following conclusions are made based on the analysis of this paper. The differential properties of film-substrate materials may contribute to the reflection effect at interfacial bonding areas when laser shockwave transmitted to the film-substrate interface. A part of compression waves may be reflected as stretching waves to effect on the interfacial bonding together for action by overcoming the interfacial bonding strength. Film and matrix were bonded by water film in model A with the weakest interfacial bonding strength, so compression waves and stretching waves would generate the most compression waves energy directing to the depth of matrix after overcoming the interfacial bonding strength. Hence, it obtained the largest peak values of piezoelectric waves and pressure waves detected by PVDF. In a similar way, the bonding strength of film and matrix bonded by double-sided tape in model $\mathrm{C}$ was the strongest and they needed to overcome the most bonding strength for acting, so it obtained the least peak values of piezoelectric waves and pressure waves detected by PVDF. Model B fell in between the two. The piezoelectric wave curve is a reflection after the comprehensive coupling of shockwaves transmitted, reflected by the interface and rebounded by the control layer. Model A had the strongest shockwave after overcoming the interfacial bonding strength and because other waves had little impact on its loading and unloading, so the piezoelectric wave curve was much smoother. At the same time, model A had the largest pressure after the pressure coupling, the most intense dynamic response, the slowest attenuation and longest time to reach the state of equilibrium. And for model $\mathrm{C}$, it had the weakest shockwave pressure after overcoming the interfacial bonding strength and it vulnerable to the impacts from the coupling of other waves during the transmission process, so it had the more abrupt piezoelectric wave, faster curve attenuation and shortest time to reach the state of equilibrium. Model B fell in between the two.

b. Delving into the influence on dynamic strain response characteristics by interfacial bonding strength

Figure 12 is the dynamic strain curve under different interfacial bonding strength and the same laser energy. It can be seen from the figure that under the same laser energy effect, model A had the largest variable quantity of stretching strain generated after the system quiesce, C had the smallest and B fell in between the two.

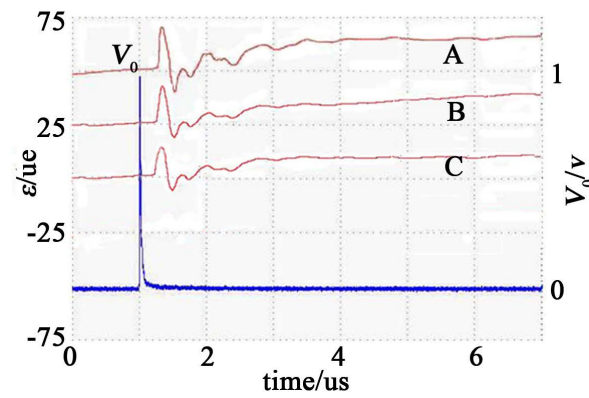

(a)

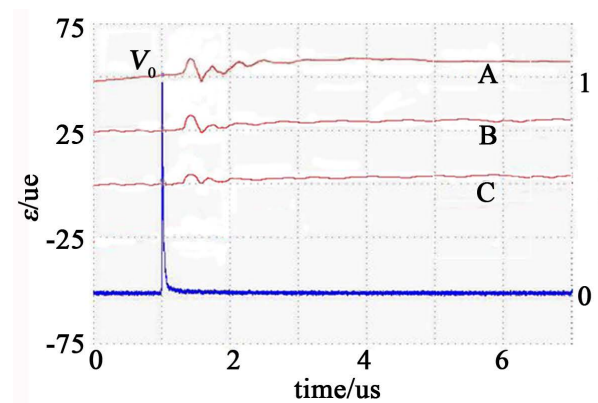

(c)

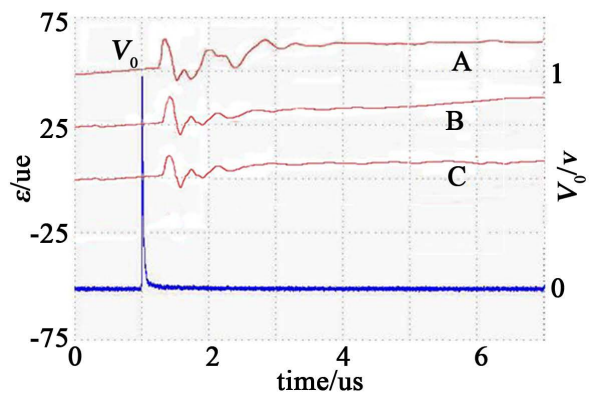

(b)

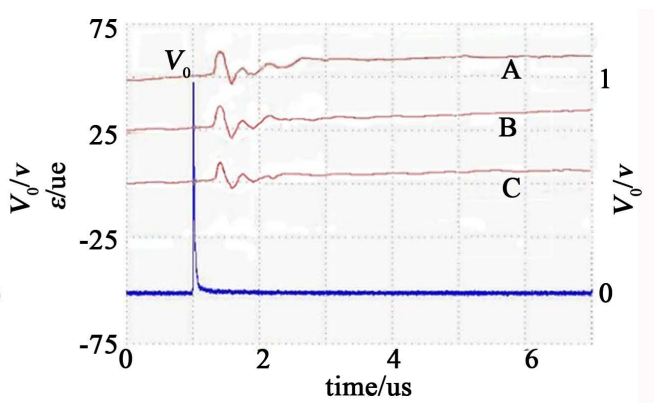

(d)

Figure 12. Dynamic strain curve under different interfacial bonding strength: (a) $1000 \mathrm{mj}$ laser energy; (b) $800 \mathrm{mj}$ laser energy; (c) 600 mj laser energy; (d) 400 mj laser energy. 
The analysis suggested that it may have something to do with energy carried by the stress wave. The interfacial bonding strength was the weakest when the water film was used to bond the film and matrix. The stress wave needed little energy to overcome the interfacial bonding strength, so more stress wave energy tightened material particles more tense. Hence, model A with the lowest energy consumption had the largest strain variable quantity, while model $\mathrm{C}$ with the highest energy consumption had the smallest strain variable quantity.

\section{Conclusions}

(1) The larger the laser energy is, the stronger the detected piezoelectric and shockwave signals, the larger the vibration amplitude and the longer for the system to reach the equilibrium state would be. The process from signal detection to the eventual system stability can be finished in microseconds.

(2) PVDF detected the stretching strain at first after the effect of laser, then the stretching strain gradually lessened and the compression strain occurred with a continuous change; the larger the laser energy is, the larger peak value of stretching strain and stretching strain of eventually generated after the system quiesce would be.

(3) Under the same laser energy effect, the model using a water film as the bonder has the largest peak values of both piezoelectric and pressure waves, smoother piezoelectric wave curve with little small-amplitude fluctuation embossment and longest time for system to reach the equilibrium state. The model using a double-sized tape as the bonder has the smallest peak values of piezoelectric and pressure waves, the least smooth piezoelectric wave curve with little small-amplitude fluctuation embossment and shortest time for dynamic process. And the model using the 502 glue as the bonder falls between the two. The model using a water film as the bonder has the largest variable quantity of stretching strain and the model using a double-sized tape as the bonder has the smallest after the system quiesce.

\section{Project Fund}

Natural Science Foundation of China (51175237, 51305050); Science \& Technology Key Special Project: “CNC Machine Tool \& Basic Manufacturing Equipment” (2013ZX04009031-4); 2014-2015 China-Russia Intergovernmental S\&T Cooperation Project by the Ministry of Science and Technology (CR17-11); International Science \& Technology Cooperation Special Project in Zhejiang Province (2013C14007); Industrial Technology R\&D Project in Wenzhou City (J20130033).

\section{References}

[1] Kandula, S.S.V., Hart-Field, C.D., Geubelle, P.H., et al. (2008) Adhesion Strength Measurement of Polymer Dielectric Interfaces Using Laser Spallation Technique. Thin Solid Films, 516, 7627-7635. http://dx.doi.org/10.1016/j.tsf.2008.05.033

[2] Feng, A.X., Xie, H.K., Zhang, Y.K., et al. (2003) Advantage Analysis of Laser Detecting Technique of Discrete Scratches for Film-Substrate Interfacial Bonding Performance. Tool Engineering, 37, 10-13.

[3] Feng, A.X. (2009) Basic Research on Laser Detecting Technique of Discrete Scratches for Film-Substrate Interfacial Bonding Performance. Jiangsu University, Zhenjiang.

[4] Cao, Y.P., Feng, A.X., Xu, C.C., et al. (2010) Laser Discrete Scratch Device for Interfacial Bonding Performance Based on the Optimization Algorithm. Chinese Journal of Lasers, 37, 2160-2164. http://dx.doi.org/10.3788/CJL20103708.2160

[5] Grupta, V., Argon, A.S., Parks, D.M., et al. (1992) Measurement of Interface Strength by a Laser Spallation Technique. Journal of the Mechanics and Physics of Solids, 40, 141-180. http://dx.doi.org/10.1016/0022-5096(92)90296-E

[6] He, P.F. and Ji, X. (1994) Theoretical Research on Development of Damages inside Composite Materials. Journal of Shanghai Jiaotong University, 28, 17-22.

[7] Shi, F. (2013) Research on Stress-Strain Characteristics of Film-Substrate System Based on Laser Detecting Technique of Discrete Scratches. Master Thesis, Jiangsu University, Zhenjiang.

[8] Shi, F., Xue, W., Feng, A.X., et al. (2013) Experiment Research on Dynamic Strain of Coating Surface Based on Laser Detecting Technique of Discrete Scratches. Applied Laser, 33, 290-293. http://dx.doi.org/10.3788/AL20133303.0290

[9] Ni, M.X., Zhou, J.Z., Yang, C.J., et al. (2006) Analysis on Intensive-Induced Residual Stress Field by Laser Shock. Laser Journal, 27, 79-83.

[10] Yu, T.X. (1990) Dynamic Plastic Response of Structure. Explosion and Shock, 10, 85-96. 
[11] Bai, S. (2006) Application of Perception Characteristics and Structural Monitoring of PVDF Dynamic Strain. Master Thesis, Harbin Institute of Technology, Harbin.

[12] Ju, D.S., Zhou, Z. and Ou, J.P. (2004) Research on Monitoring Cracks of Metal Components Based on PVDF. Piezoelectrics \& Acoustooptics, 26, 245-248.

[13] Wang, L.L. (2001) New Development of Researches on Dynamic Response of Structure and Materials under Explosion and Shock Load. Explosion and Shock, 21, 81-88. 